\title{
Dietas populares: Ayuno intermitente ${ }^{1}$
}

\author{
Michelle Yavelow, Daniela Rivero-Mendoza, y Wendy J. Dahl²
}

La pérdida de peso puede ser un desafío. Comenzar una dieta restrictiva puede ser agotador emocionalmente y difícil de cumplir (Harris et al. 2018). ¿Hay alguna forma más efectiva de perder peso? ¿El momento y la frecuencia de las comidas ayudan a perder peso? Esta publicación describe los beneficios y riesgos del ayuno intermitente para bajar de peso.

\section{¿Qué es el ayuno intermitente?}

El ayuno es evitar completamente la comida o la bebida. El ayuno se ha practicado para las celebraciones religiosas a lo largo de la historia. Por ejemplo, durante el Ramadán, los musulmanes ayunan desde el amanecer hasta el atardecer todos los días durante 30 días. El ayuno intermitente incluye una variedad de patrones de alimentación donde el ayuno ocurre durante períodos de tiempo específicos de forma repetitiva (Anton et al. 2018). Recientemente, el ayuno intermitente se ha considerado un medio para promover la pérdida de peso (Cho et al. 2019, Harris et al. 2018, Welton et al. 2020). Hay una variedad de patrones de ayuno intermitente. Algunos de los patrones comunes se describen a continuación en la Tabla 1.
Tabla 1. Patrones alimentarios de ayuno intermitente.

\begin{tabular}{|l|l|}
\hline Patrón de ayuno & Explicación del patrón de ayuno \\
\hline $\begin{array}{l}\text { Alimentación restringida } \\
\text { en tiempo }\end{array}$ & Ayuno de 8 a 16 horas cada día \\
\hline Ayuno de días alternados & $\begin{array}{l}\text { Ayuno durante } 24 \text { horas seguido } \\
\text { un día completo de alimentación } \\
\text { restricciones }\end{array}$ \\
\hline $\begin{array}{l}\text { Ayuno modificado de } \\
\text { días alternados }\end{array}$ & $\begin{array}{l}\text { Permite el 25\% del requerimiento } \\
\text { calórico en el día de ayuno }\end{array}$ \\
\hline Ayuno periódico & $\begin{array}{l}\text { Ayuno durante } 1 \text { o } 2 \text { días (puede } \\
\text { permitir } 25 \% \text { de las necesidades } \\
\text { calóricas) y alimentación sin } \\
\text { restricciones el resto de la semana }\end{array}$ \\
\hline Adaptado de Anton et al. (2018), Patterson et al. (2015)
\end{tabular}

La pérdida de peso es un objetivo importante para algunas personas con sobrepeso u obesidad. Si tiene sobrepeso u obesidad, perder peso puede disminuir las probabilidades de desarrollar enfermedades crónicas, como enfermedades cardiovasculares y diabetes tipo 2 (Kritchevsky et al. 2015). Las estrategias de pérdida de peso a menudo incluyen la restricción continua de calorías, una disminución en la cantidad de calorías que una persona come cada día. Los estudios de investigación muestran que el ayuno intermitente no es más efectivo para perder peso que la restricción continua de calorías (Cioffi et al. 2018, Harris et al. 2018, Roman et al. 2019). Sin embargo, en comparación con el

1. Este documento, FSHN20-47s, es uno de una serie de publicaciones del Food Science and Human Nutrition, Servicio de Extensión Cooperativa de la Florida, Instituto de Alimentos y Ciencias Agrícolas, Universidad de la Florida. (UF/IFAS). Fecha de primera publicación: octubre 2020. Visite nuestro sitio web EDIS en https://edis.ifas.ufl.edu.

2. Michelle Yavelow, former graduate student; Daniela Rivero Mendoza, Extension and research coordinator; and Wendy Dahl, associate professor, Food Science and Human Nutrition Department; UF/IFAS Extension, Gainesville, FL 32611.

The Institute of Food and Agricultural Sciences (IFAS) is an Equal Opportunity Institution authorized to provide research, educational information and other services

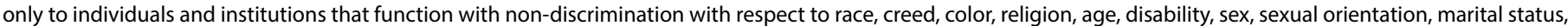

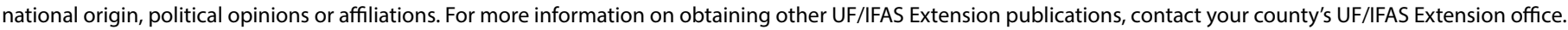
U.S. Department of Agriculture, UF/IFAS Extension Service, University of Florida, IFAS, Florida A \& M University Cooperative Extension Program, and Boards of County Commissioners Cooperating. Nick T. Place, dean for UF/IFAS Extension. 
consumo de una dieta habitual sin restricciones, el ayuno intermitente produce una pérdida de peso moderada de menos de 10 libras (Harris et al. 2018).

En individuos con diabetes tipo 2, el ayuno durante 2 días a la semana (500-600 kcal / día) mejoró el peso, las mediciones de glucosa en sangre y la calidad de vida (Corley et al. 2018). Sin embargo, para las personas con diabetes tipo 2 que toman medicamentos para reducir la glucosa, el ayuno de cualquier tipo aumentó la tasa de hipoglucemia (bajo nivel de azúcar en la sangre) (Corley et al. 2018), que, si no se trata, puede provocar mareos, debilidad, convulsiones, pérdida de conciencia e incluso la muerte. Se necesita educación adecuada y manejo de medicamentos por parte de un profesional de la salud para reducir el riesgo de eventos hipoglucémicos mientras se practica el ayuno intermitente (Corley et al. 2018).

En adultos con sobrepeso u obesidad o aquellos que no tienen diabetes, el ayuno intermitente no mejora la glucemia en ayunas, la resistencia a la insulina o el colesterol LDL (malo) (Cioffi et al. 2018, Harris et al. 2018). Sin embargo, una revisión de estudios informó una mejor calidad de vida con ayuno intermitente, en comparación con la restricción de energía continua (Harris et al. 2018). Revisiones recientes de estudios encontraron que el ayuno intermitente y las dietas continuas restringidas en calorías son más efectivas para la mejora de la mayoría de los marcadores de salud cardiovascular, como el colesterol en la sangre, en comparación con la no dieta (Meng et al. 2020). Además, se ha informado una reducción leve pero significativa en la insulina en ayunas para el ayuno intermitente, en comparación con la restricción calórica continua (Cioffi et al. 2018).

Los patrones de ayuno intermitente con períodos de alimentación de 6 o 12 horas (es decir, períodos de ayuno de 12 a 18 horas) se compararon en hombres con prediabetes. El estudio fue diseñado para proporcionar a los participantes suficientes alimentos para mantener su peso corporal. El período temprano de alimentación con restricción de tiempo mejoró la sensibilidad a la insulina y la presión arterial (Sutton et al. 2018). Además, el período temprano de alimentación con restricción de tiempo redujo el apetito por la noche, a pesar de la mayor duración del ayuno (Sutton et al. 2018).

Durante la pérdida de peso, es deseable perder grasa corporal mientras se mantiene la masa magra, es decir, la masa muscular. Las revisiones de los estudios de investigación muestran que el ayuno intermitente no conserva la masa magra más que las dietas continuas con restricción calórica
(Clifton 2017, Harris et al. 2018) e incluso puede conducir a una disminución de la masa magra (Roman et al. 2019). Dos revisiones de estudios de investigación mostraron que la pérdida de masa grasa con el ayuno intermitente es similar a las dietas continuas restringidas en calorías (Cho et al. 2019), mientras que una revisión de adultos con sobrepeso y obesidad informó un efecto beneficioso con el ayuno intermitente (Harris et al. 2018). Sin embargo, el nivel de adiponectina, una hormona asociada con la descomposición de la grasa corporal y la sensibilidad a la insulina puede aumentar con el ayuno intermitente (Cho et al. 2019).

Efectos metabólicos del ayuno
Cuando come una comida, su cuerpo comienza a descomponer
estos alimentos en nutrientes más pequeños que su cuerpo
puede usar. Su cuerpo normalmente digiere los carbohidratos
y usa glucosa, un azúcar, como fuente de energía. El cuerpo
humano requiere 130 gramos de glucosa por día para mantener
nuestro cerebro y sistema nervioso funcionando bien (Institute
of Medicine and Food and Nutrition Board 2005). Sin embargo,
ayunar o consumir una ingesta muy baja de carbohidratos puede
evitar que el cuerpo obtenga suficiente glucosa para tener energía
(Fedorovich, Voronina, and Waseem 2018 ). En su lugar, el cuerpo
comenzará a quemar grasas como fuente de energía durante
el ayuno. Este cambio ocurrirá de 12 a 32 horas después de que
comience a ayunar, dependiendo de la cantidad de carbohidratos
almacenados en su cuerpo. Este cambio ocurrirá más rápido si hace
ejercicio durante un período de ayuno. Es importante destacar que
algunos rituales de ayuno no alcanzan el punto en que el cuerpo
comienza a quemar grasas. Por ejemplo, en 2019 , la duración del
ayuno del Ramadán (una tradición islámica que incluye el ayuno
durante el día) fue de 11 horas a 20 horas dependiendo de la latitud,
por lo que es posible que algunos de ellos no hayan alcanzado el
cambio (Al Jazeera News 2010 ).

\section{Potenciales riesgos de salud}

Se han realizado pocos estudios para determinar la seguridad a largo plazo del ayuno intermitente (Liu et al. 2020). Es importante destacar que existen riesgos para ciertas poblaciones, especialmente si las personas no son seguidas cuidadosamente por un profesional de la salud. Lo más importante es que el ayuno intermitente puede provocar hipoglucemia en personas que tienen diabetes tipo 2 y están tomando medicamentos para reducir la glucosa (Corley et al. 2018).

El ayuno intermitente puede ser difícil de cumplir para algunas personas (Harris et al. 2018), pero con la variedad de métodos enumerados en el cuadro anterior, puede encontrar un patrón que funcione con su estilo de vida. A algunos profesionales de la salud les preocupa que el ayuno intermitente pueda alentar la alimentación desordenada (Liu et al. 2020). En consecuencia, la mayoría de los estudios de investigación han excluido a personas con antecedentes médicos de trastornos alimentarios y 
afecciones psiquiátricas (Liu et al. 2020). Si actualmente tiene o le diagnosticaron estas afecciones, el ayuno intermitente puede no ser apropiado para usted.

\section{Resumen}

Los estudios han comparado el ayuno intermitente con comer menos todos los días y encontraron que ambos resultan en una pérdida de peso similar. Existe alguna evidencia de que el ayuno intermitente puede mejorar la sensibilidad a la insulina y la presión arterial independientemente de la pérdida de peso. Sin embargo, existen riesgos relacionados con el ayuno intermitente. El ayuno representa un riesgo serio de hipoglucemia en personas con diabetes tipo 2 que toman medicamentos para bajar la glucosa. En aquellos que actualmente están diagnosticados o han sido previamente diagnosticados con un desorden de alimentación, el ayuno intermitente puede no ser recomendado. Además, como cualquier otro patrón de alimentación en el que la ingesta de calorías puede ser limitada, la adherencia al ayuno intermitente puede ser un desafío. La fuerza de la evidencia con respecto a los efectos del ayuno intermitente sobre el peso y los factores de riesgo cardio metabólico es débil en comparación con la evidencia fuerte y consistente de los beneficios para la salud del patrón dietético mediterráneo (Dinu et al. 2020).

Nota: Es importante hablar con su proveedor de atención médica o nutricionista dietista registrado antes de realizar cambios en su dieta.

\section{Referencias}

Al Jazeera News. 2010. "Ramadan 2020: Fasting Hours around the World." Accessed June 15, 2020. https://www. aljazeera.com/indepth/interactive/2017/05/ramadan2017-fasting-hours-world-170511102015757.html.

Anton, Stephen D., Keelin Moehl, William T. Donahoo, Krisztina Marosi, Stephanie A. Lee, Arch G. Mainous III, Christiaan Leeuwenburgh, and Mark P. Mattson. 2018. "Flipping the Metabolic Switch: Understanding and Applying the Health Benefits of Fasting." Obesity 26 (2): 254-268. https://doi.org/10.1002/oby.22065.

Cho, Yongin, Namki Hong, Kyung-won Kim, Sung joon Cho, Minyoung Lee, Yeon-hee Lee, Yong-ho Lee, Eun Seok Kang, Bong-Soo Cha, and Byung-Wan Lee. 2019. "The Effectiveness of Intermittent Fasting to Reduce Body Mass Index and Glucose Metabolism: A Systematic Review and Meta-analysis." Journal of Clinical Medicine 8 (10): 1645. https://doi.org/10.3390/jcm8101645.
Cioffi, Iolanda, Andrea Evangelista, Valentina Ponzo, Giovannino Ciccone, Laura Soldati, Lidia Santarpia, Franco Contaldo, Fabrizio Pasanisi, Ezio Ghigo, and Simona Bo. 2018. "Intermittent versus Continuous Energy Restriction on Weight Loss and Cardiometabolic Outcomes: A Systematic Review and Meta-analysis of Randomized Controlled Trials." Journal of Translational Medicine 16 (1): 371. https:// doi.org/10.1186/s12967-018-1748-4.

Clifton, Peter. 2017. "Assessing the Evidence for Weight Loss Strategies in People with and without Type 2 Diabetes." World Journal of Diabetes 8 (10): 440-454. https://doi. org/10.4239/wjd.v8.i10.440.

Corley, B. T., R. W. Carroll, R. M. Hall, M. Weatherall, A. Parry-Strong, and J. D. Krebs. 2018. "Intermittent Fasting in Type 2 Diabetes Mellitus and the Risk of Hypoglycaemia: A Randomized Controlled Trial." Diabetic Medicine 35 (5): 588-594. https://doi.org/10.1111/dme.13595.

Dinu, Monica, Giuditta Pagliai, Donato Angelino, Alice Rosi, Margherita Dall'Asta, Letizia Bresciani, Cinzia Ferraris, Monica Guglielmetti, Justyna Godos, Cristian Del Bo, Daniele Nucci, Erika Meroni, Linda Landini, Daniela Martini, Francesco Sofi, and The Working Group "Young Members" of the Italian Society of Human Nutrition. 2020. "Effects of Popular Diets on Anthropometric and Cardiometabolic Parameters: An Umbrella Review of Meta-analyses of Randomized Controlled Trials." Advances in Nutrition 11 (4): 815-833. https://doi.org/10.1093/ advances/nmaa006.

Fedorovich, Sergei V., Polina P. Voronina, and Tatyana V. Waseem. 2018. "Ketogenic Diet versus Ketoacidosis: What Determines the Influence of Ketone Bodies on Neurons?" Neural Regeneration Research 13 (12): 2060-2063. https:// doi.org/10.4103/1673-5374.241442.

Harris, Leanne, Sharon Hamilton, Liane B. Azevedo, Joan Olajide, Caroline De Brún, Gillian Waller, Vicki Whittaker, Tracey Sharp, Mike Lean, Catherine Hankey, and Louisa Ells. 2018. "Intermittent Fasting Interventions for Treatment of Overweight and Obesity in Adults: A Systematic Review and Meta-analysis." JBI Evidence Synthesis 16 (2): 507-547. https://doi.org/10.11124/jbisrir-2016-003248.

Horne, B. D., M. M. Grajower, and J. L. Anderson. 2020. "Limited Evidence for the Health Effects and Safety of Intermittent Fasting among Patients with Type 2 Diabetes." JAMA 324 (4): 341-342. https://doi.org/10.1001/ jama.2020.3908. 
Institute of Medicine, and Food and Nutrition Board. 2005. Dietary Reference Intakes for Energy, Carbohydrate, Fiber, Fat, Fatty Acids, Cholesterol, Protein, and Amino Acids. Washington, D.C.: The National Academies Press.

Kritchevsky, Stephen B., Kristen M. Beavers, Michael E. Miller, M. Kyla Shea, Denise K. Houston, Dalane W. Kitzman, and Barbara J. Nicklas. 2015. "Intentional Weight Loss and All-Cause Mortality: A Meta-analysis of Randomized Clinical Trials." PloS one 10 (3): e0121993-e0121993. https://doi.org/10.1371/journal.pone.0121993.

Liu, Kai, Bo Liu, and Leonie K Heilbronn. 2020. "Intermittent Fasting: What Questions Should We Be Asking?" Physiology \& Behavior 218:112827.

Meng, Haiyan, Lei Zhu, Hamed Kord-Varkaneh, Heitor O. Santos, Grant M. Tinsley, and Peng Fu. 2020. "Effects of Intermittent Fasting and Energy-Restricted Diets on Lipid Profile: A Systematic Review and Meta-analysis." Nutrition 77:110801. https://doi.org/10.1016/j.nut.2020.110801.

Patterson, Ruth E., Gail A. Laughlin, Andrea Z. LaCroix, Sheri J. Hartman, Loki Natarajan, Carolyn M. Senger, María Elena Martínez, Adriana Villaseñor, Dorothy D. Sears, Catherine R. Marinac, and Linda C. Gallo. 2015. "Intermittent Fasting and Human Metabolic Health." Journal of the Academy of Nutrition and Dietetics 115 (8): 1203-1212. https://doi.org/10.1016/j.jand.2015.02.018.

Roman, Yuani M., Mariah C. Dominguez, Tommy M. Easow, Vinay Pasupuleti, C. Michael White, and Adrian V. Hernandez. 2019. "Effects of Intermittent versus Continuous Dieting on Weight and Body Composition in Obese and Overweight People: A Systematic Review and Meta-analysis of Randomized Controlled Trials." International Journal of Obesity 43 (10): 2017-2027. https:// doi.org/10.1038/s41366-018-0204-0.

Sutton, Elizabeth F., Robbie Beyl, Kate S. Early, William T. Cefalu, Eric Ravussin, and Courtney M. Peterson. 2018. "Early Time-Restricted Feeding Improves Insulin Sensitivity, Blood Pressure, and Oxidative Stress Even without Weight Loss in Men with Prediabetes." Cell Metabolism 27 (6): 1212-1221.e3. https://doi.org/10.1016/j. cmet.2018.04.010.

Welton, Stephanie, Robert Minty, Teresa O'Driscoll, Hannah Willms, Denise Poirier, Sharen Madden, and Len Kelly. 2020. "Intermittent Fasting and Weight Loss." Canadian Family Physician 66 (2): 117-125. 